\title{
Joint Two-Dimensional Ambiguity Resolving Based on Space-Time Filtering for MIMO-SAR
}

\author{
Ping-ping Huang, ${ }^{1}$ Hua-sheng Li, ${ }^{1}$ Sheng Zhang, ${ }^{2}$ and Guang-Cai Sun ${ }^{2}$ \\ ${ }^{1}$ College of Information Engineering, Inner Mongolia University of Technology, Hohhot 010051, China \\ ${ }^{2}$ The National Key Laboratory of Radar Signal Processing, Xidian University, Xian 710071, China \\ Correspondence should be addressed to Ping-ping Huang; cimhwangpp@163.com
}

Received 16 March 2014; Revised 17 June 2014; Accepted 1 July 2014; Published 12 August 2014

Academic Editor: Shengqi Zhu

Copyright (c) 2014 Ping-ping Huang et al. This is an open access article distributed under the Creative Commons Attribution License, which permits unrestricted use, distribution, and reproduction in any medium, provided the original work is properly cited.

In order to resolve the range and azimuth ambiguity in MIMO-SAR imaging, this paper proposed a joint two-dimensional ambiguity resolving method based on space-time filtering, which according to the ambiguity in different subscene is relative to different nearest echo range, and different azimuth time is relative to different instantaneous incidence angle, to make twodimensional space-time steering vector for resolving ambiguity of the echo, which also can finish the imaging processing at the same time. Simulation results validate the proposed processing approach for the MIMO-SAR system.

\section{Introduction}

Multiple-input and multiple-output spaceborne synthetic aperture radar (MIMO-SAR), as a high resolution and wide swath microwave remote sense system [1-5], is proved to be an extremely useful surveillance tool for moving target indication, wide swath imaging, and three-dimensional imaging.

ScanSAR also can obtain wide unambiguous swath coverage at the cost of a degraded azimuth resolution [6]. Multichannel line arrays can be arranged by range or azimuth [7], for the range multichannel can obtain wide swath coverage, utilizing digital beamforming to resolve range ambiguity, but there is still blind zone $[8,9]$. With the emergence of techniques such as displaced phase center [10] and multiple aperture reconstruction [11, 12], a number of displaced azimuth subapertures are used to receive echoes in one pulse repetition interval (PRI), thereby increasing the effective azimuth sampling rate without changing the pulse repetition frequency (PRF). In this way, one can lower the requirement on PRF to achieve wider swath imaging without impairing azimuth resolution; in other words, one can broaden the Doppler spectrum to obtain finer azimuth resolution without reducing the swath coverage.

To obtain wide swath coverage and high resolution, one can utilize two-dimensional array to transmit and receive
SAR signals and then resolve the range and azimuth ambiguity [13]. However, this method makes range point target downlooking angle as range steer vector in approximate treatment and does not consider the changing of the downlooking angle with the azimuth time.

In this paper, a joint two-dimensional ambiguity resolving method for MIMO-SAR is proposed, which uses deferent targets relative to deferent range as steer vector which is independent of the downlooking angle. Firstly, the azimuth ambiguity is resolved. Then, the range ambiguity resolving and azimuth match filtering are performed instantaneously to get target image, which makes the imaging algorithms simpler.

This paper is organized as follows. Section 2 reviews the signal model of MIMO-SAR. The two-dimensional ambiguity resolving algorithm is analyzed in Section 3. Section 4 gives the simulations and validates the proposed processing approach for the MIMO-SAR system. Finally, Section 5 concludes this paper.

\section{MIMO-SAR System Mode and Signal Model}

Figure 1 presents the geometry of a multichannel SAR. It supposed that the whole antenna aperture was divided into 


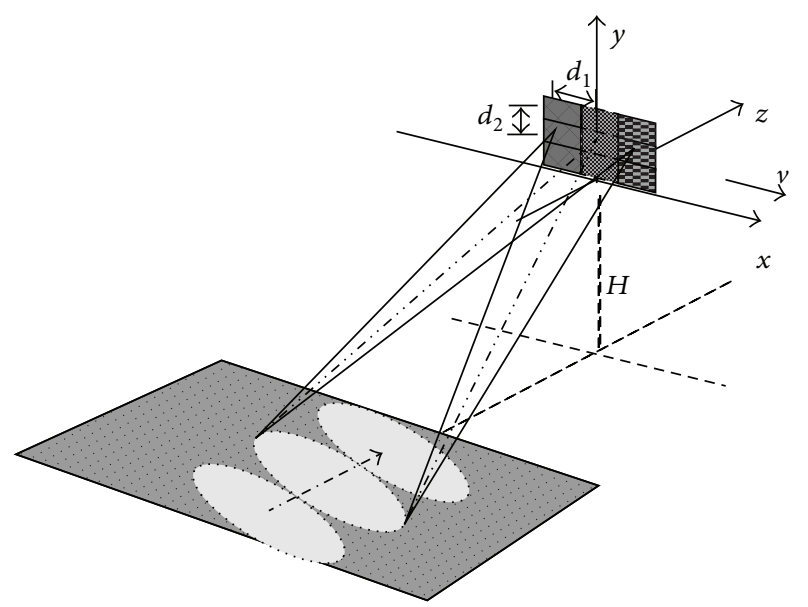

FIgURE 1: MIMO-SAR model.

$P \times Q$ subapertures, where $P$ is the number of azimuth subapertures and its length is $d_{1}$. $Q$ is the number of azimuth subapertures and its height is $d_{2}$. We denoted the $p$ th column and the $q$ th row as $X_{p}=X_{0}+(p-1) d_{1}, p=1, \ldots, P$, with $X_{0}$ the azimuth beginning coordinate $\left(X_{p}, Y_{q}, Z\right)$, and $Y_{q}=Y_{0}+(q-1) d_{2}, q=1, \ldots, Q$, with $Y_{0}$ the range beginning coordinate.

Use the subcaptures on the same column range dimension transmit signals at the same time; through adjusting phase weighting to control the wave beam, the center of transmit wave beam is $Y_{q}^{\prime}=\left(Y_{q}+Y_{q_{0}}\right) / 2, Y_{q_{0}}=Y_{0}+d_{2}(Q-1) / 2$, and the whole antenna receives the echo signal. According to the principle of equivalent phase center, this mode can be known as each equivalent phase center transmitting and receiving itself. The equivalent phase centers located at $\left(X_{p}^{\prime}, Y_{p}^{\prime}, Z\right)$, where $X_{p}^{\prime}=\left(X_{0}+X_{p}\right) / 2$ and $Y_{p}^{\prime}=\left(Y_{q}+Y_{q_{0}}\right) / 2$. The deferent range subapertures light the deferent subswaths from far to nearby adjusting phase weightings. The ellipses in Figure 1 are the subswathes.

Assume that, during the $L$ th pulse period, the first range dimension subapertures transmit signal in the same time and make the wave beam light the Lth subswath through controlling phase weighting. The transmitting signal can be expressed as follows:

$$
\begin{aligned}
s\left(t_{k}\right)= & \frac{\sin \left(\pi Q d_{2}\left(\sin \theta_{l}-\sin \theta\right) / 2 \lambda\right)}{\sin \left(\pi d_{2}\left(\sin \theta_{l}-\sin \theta\right) / 2 \lambda\right)} \\
& \times \operatorname{rect}\left(\frac{\left(t_{k}-\Delta T\right)}{T_{p_{l}}}\right) \\
& \times \exp \left(j 2 \pi f_{c}\left(t-\Delta T_{l}\right)+j \pi \gamma\left(t_{k}-\Delta T_{l}\right)^{2}\right),
\end{aligned}
$$

where $\theta_{l}$ is the downlooking angle of the center line of th subswath, $f_{c}$ is the carrier frequency, $\lambda=c / f_{c}$ is the wavelength, $\Delta T_{l}=T_{p_{1}}+\cdots+T_{p_{l-1}}$ is the delay time of the lth subpulse, $t=t_{k}+m T_{r}$ is the whole time, $t_{k}$ is the fast time, $m$ is a integer, $T_{r}$ is pulse repetition interval, and $\gamma$ is the chirp rate.
The baseband signal received by the $p$ th row and the $q$ th column subaperture was

$$
\begin{aligned}
s_{p q}\left(t_{k}, t_{m}\right)= & \sum_{l=1}^{L} A\left(\theta_{l}\right) \operatorname{rect}\left(\frac{\left(t_{k}-\Delta T_{l}-2 R_{p q, l}\left(t_{m}\right) / c\right)}{T_{p_{l}}}\right) \\
& \times \operatorname{rect}\left(\frac{\left(X_{p}^{\prime}+x-x_{l}\right)}{L_{a}}\right) \\
& \times \exp \left(j 2 \pi f_{c}\left(-\Delta T_{l}-\frac{2 R_{p q, l}\left(t_{m}\right)}{c}\right)\right) \\
& \times \exp \left(j \pi \gamma\left(t_{k}-\Delta T_{l}-\frac{2 R_{p q, l}\left(t_{m}\right)}{c}\right)^{2}\right)
\end{aligned}
$$

where $A\left(\theta_{l}\right)=\sin \left(\pi Q d_{2}\left(\sin \theta_{l}-\sin \theta\right) / 2 \lambda\right) / \sin \left(\pi d_{2}\left(\sin \theta_{l}-\right.\right.$ $\sin \theta) / 2 \lambda), t_{m}=k T_{r}$ is slow time, $k$ is a integer, and the value scope of $l$ is from 1 to $L$, corresponding to the swath from far to near. $x=v t_{m}, v$ is the flat velocity, and $L_{a}$ is the length of synthetic aperture. $R_{p q, l}\left(t_{m}\right)=$ $\sqrt{\left(X_{p}^{\prime}+v t_{m}-x_{l}\right)^{2}+\left(Y_{p}^{\prime}-y_{l}\right)^{2}+H^{2}}$ is the instantaneous slant range, $\left(x_{l}, y_{l}, z_{l}\right)$ is the coordinates of one scatter point in the $L$ th scene, and $H=Z_{0}-z_{l}$. Assume that scene is plane, $z_{l}=0$.

Performing the range match filtering to (2), we can gain

$$
\begin{aligned}
s_{p q}\left(t_{k}, t_{m}\right) \approx & \sum_{l=1}^{L} A^{\prime}\left(\theta_{l}\right) \sin c\left(t_{k}-\Delta T_{l}-\frac{2 R_{l}\left(t_{m}\right)}{c}\right) \\
& \times \operatorname{rect}\left(\frac{X_{p}^{\prime}+x-x_{l}}{L_{a}}\right) \exp \left(-j \frac{4 \pi}{\lambda} R_{p q, l}\left(t_{m}\right)\right),
\end{aligned}
$$

where $A^{\prime}\left(\theta_{l}\right)=A\left(\theta_{l}\right) \exp \left(-j 2 \pi f_{c} \Delta T_{l}\right), R_{l}\left(t_{m}\right)=$ $\sqrt{\left(X_{0}+v t_{m}-x_{l}\right)^{2}+\left(Y_{0}-y_{l}\right)^{2}+H^{2}}$ is the instantaneous distance from the scatter point on the $l$ th subswath to $\left(X_{0}, Y_{0}, Z_{0}\right)$.

As we know, the MIMO-SAR can be considered as a space signal sampling which should satisfy the sampling equation as follows:

$$
d \leq \frac{\lambda}{2}
$$

where $d$ denotes the position change of antenna element and $\lambda$ denotes the wavelength of the signal.

So the time delay should be

$$
T_{d}=\frac{d}{c} \leq \frac{\lambda}{2 c}=\frac{1}{2 f_{c}},
$$

where $f_{c}$ denotes the carrier frequency. 


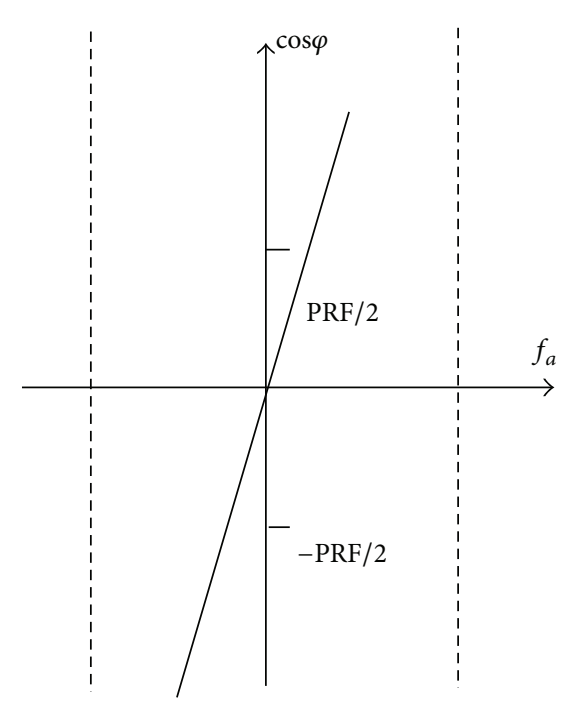

(a) The Doppler is not ambiguity

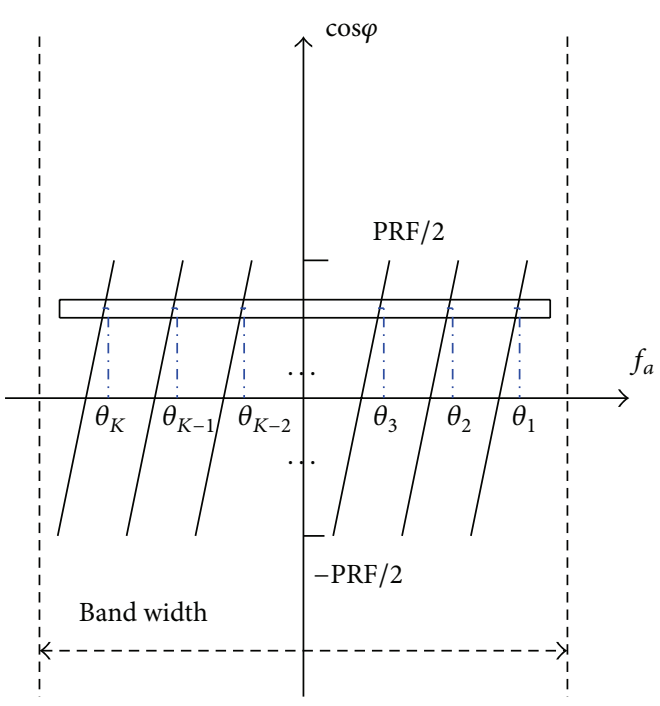

(b) The Doppler is ambiguity

FIgURE 2: The relationship between cosine of cone angle and Doppler.

And the time resolution can be calculated by

$$
T_{r}=\frac{1}{2 B}
$$

where $B$ denotes the bandwidth.

In a narrow band system $f_{c}$ is much larger than $B$, so we can get $T_{d}$ much smaller than $T_{r}$, which means that the time delay made by the position change of antenna element is very small compared with the time resolution.

The time delay made by the position change of antenna element can be ignored, which is reasonable because the distance between the elements is very small.

This system uses low PRF to get wide swath, but the low PRF can cause azimuth Doppler ambiguity; Figure 2 illuminates this.

After azimuth fast Fourier transforms (FFT), we can gain the Doppler ambiguity signal $s_{p q}\left(t_{k}, f_{a}\right)$ :

$$
\begin{aligned}
s_{p q}\left(t_{k}, f_{a}\right)= & \sum_{l=1}^{L} \sum_{k=1}^{K} A^{\prime}\left(\theta_{l}\right) \sin c\left(t_{k}-\Delta T_{l}-\frac{2 R_{l}\left(f_{a}\right)}{c}\right) \\
& \times \operatorname{rect}\left(\frac{f_{a} R_{B 1}}{\sqrt{f_{a m}^{2}-f_{a}^{2}}}\right) \exp \left(-j \frac{2 \pi f_{a} x_{l}}{v}\right) \\
& \times \exp \left(-j \frac{2 \pi R_{B q}}{v} \sqrt{f_{a m}^{2}-f_{a}^{2}}\right) \\
& \times \exp \left(j \frac{4 \pi X_{p}^{\prime} \cos \varphi_{k}\left(f_{a}\right)}{\lambda}\right),
\end{aligned}
$$

where $f_{a} \in(-\mathrm{PRF} / 2, \mathrm{PRF} / 2), R_{B q}=\sqrt{\left(Y_{q}-y_{1}\right)^{2}+H^{2}}$ is the vertical distance form scatter point $\left(x_{1}, y_{1}, z_{1}\right)$ to flight track,
$R_{p q, 1}\left(f_{a}\right)$ is the instantaneous distance of scatter point, $\varphi_{k}$ is the angle of instantaneous slant distance and flight track, and $\cos \varphi_{k}\left(f_{a}\right)=f_{a} / f_{a m}, f_{a m}=2 v / \lambda$.

When $R_{1}=c T_{p_{1}} / 2+R_{2}=\cdots=c\left(T_{p_{1}}+\cdots+T_{p_{L-1}}\right) / 2+R_{L}$ is satisfied, $L$ different scatter points on $L$ subswathes can make aliasing and then cause range ambiguity. But the different scatter points are corresponding to different downlooking angles, as shown in Figure 3.

According to the forgoing analysis, if the scatter instantaneous slant distance satisfies the preceding equation, (7) can be shown as

$$
\begin{aligned}
s_{p q}\left(t_{k}, f_{a}\right)= & \sum_{l=1}^{L} \sum_{k=1}^{K} A^{\prime}\left(\theta_{l}\right) \sin c\left(t_{k}-\frac{2 R_{1}}{c}\right) \\
& \times \operatorname{rect}\left(\frac{f_{a} R_{B 1}}{\sqrt{f_{a m}^{2}-f_{a}^{2}}}\right) \exp \left(-j \frac{2 \pi f_{a} x_{l}}{v}\right) \\
& \times \exp \left(-j \frac{2 \pi R_{B q}}{v} \sqrt{f_{a m}^{2}-f_{a}^{2}}\right) \\
& \times \exp \left(-j \frac{4 \pi X_{p}^{\prime} \cos \varphi_{k}\left(f_{a}\right)}{\lambda}\right),
\end{aligned}
$$

where $\exp \left(-j\left(2 \pi R_{B q} / v\right) \sqrt{f_{a m}^{2}-f_{a}^{2}}\right)$ and $\exp \left(j\left(4 \pi X_{p}^{\prime} \cos \varphi_{k}\right.\right.$ $\left.\left(f_{a}\right) / \lambda\right)$ ) are caused by the position of azimuth and range subapertures, and they are the steer vector for resolving ambiguity. One can know that there is the azimuth frequency in the range steer vector, so one needs to resolve the azimuth ambiguity firstly and then resolve the range ambiguity. 


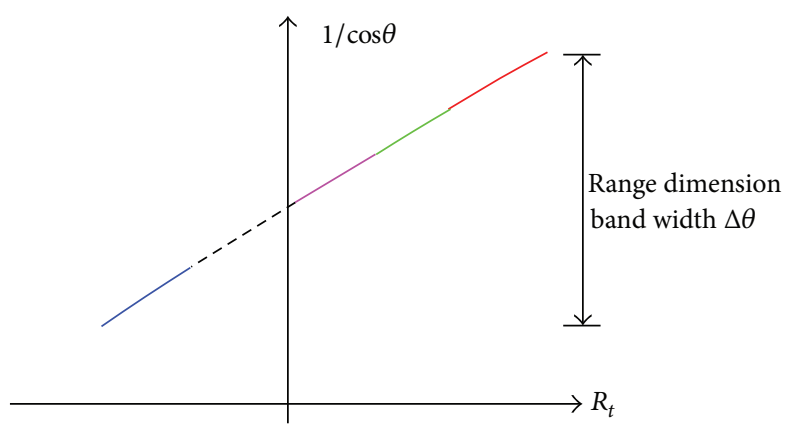

(a) The range is not ambiguity

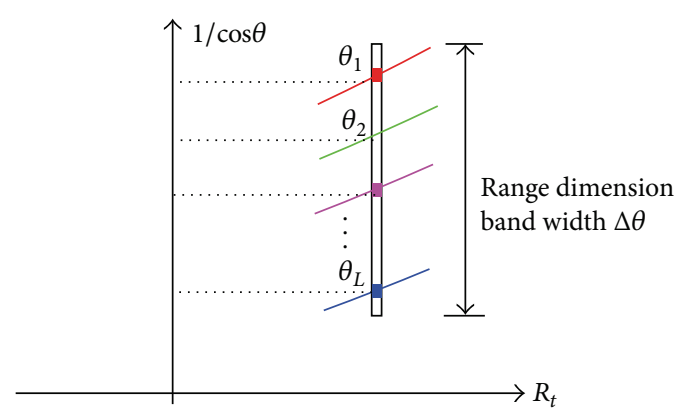

(b) The range is ambiguity

FIGURE 3: The relationship between downlooking angle and instantaneous slant distance.

\section{Two-Dimensional Ambiguity Resolving}

3.1. Resolving Azimuth Ambiguity. Because (8) is range and Doppler ambiguity signal, we express the matrix vector $\mathbf{z}_{a}\left(\varphi_{k}\right)$ as

$$
\begin{aligned}
& \mathbf{z}_{a}\left(\varphi_{k}\right) \\
& \quad=\left[\exp \left(\frac{j 4 \pi X_{1}^{\prime} \cos \varphi_{k}}{\lambda}\right), \ldots, \exp \left(\frac{j 4 \pi X_{P}^{\prime} \cos \varphi_{k}}{\lambda}\right)\right]^{T} .
\end{aligned}
$$

In this paper, we use joint 2-dimensional method to resolve ambiguity based on static weighting vector. It is assumed that the vector matrix is $\mathbf{W}_{P \times K}$ and the $n$th column weighting vector is shown as

$$
\mathbf{W}_{n}=\left[w_{1, n}, \ldots, w_{p, n}, \ldots, w_{P, n}\right]^{T} .
$$

It means that the corresponding downlooking angle and azimuth angle position is 1 and other ambiguities are zero. Consider

$$
\mathbf{W}_{n}^{H} z_{a}=\mathbf{H}_{n}
$$

where ()$^{H}$ expresses matrix conjugate transpose operator and $\mathbf{H}_{n}=\left[h_{1 n}, \ldots, h_{K n}\right]^{T}, n=1, \ldots, K$, when $k=n, h_{k n}=1$. Consider

$$
\mathbf{W}_{n}=\left(\mathbf{H}_{n} z_{a}^{+}\right)^{H}
$$

where ()$^{+}$denotes the pseudoinverse operation, $z_{a}^{+}=$ $\operatorname{pinv}\left(z_{a}\right)$. Consider

$$
\begin{aligned}
\mathbf{S}= & {\left[s_{11}, \ldots, s_{P 1}, s_{12}, \ldots, s_{P 2}, \ldots, s_{1 Q}, \ldots, s_{P Q}\right]^{T} } \\
= & \operatorname{sinc}\left(t_{k}-\frac{2 R_{1}}{c}\right) \operatorname{rect}\left(\frac{f_{a} R_{B 1}}{\sqrt{f_{a m}^{2}-f_{a}^{2}}}\right) \exp \left(-j \frac{2 \pi f_{a} x_{l}}{v}\right) \\
& \times \exp \left(-j \frac{2 \pi R_{B q} \sqrt{f_{a m}^{2}-f_{a}^{2}}}{v}\right) z_{a} .
\end{aligned}
$$

Using the weighting vector to resolve ambiguity, the following is gained:

$$
\begin{aligned}
& \mathbf{S}^{\prime}=\mathbf{W}_{n}^{H} \mathbf{S}=\operatorname{sinc}\left(t_{k}-\frac{2 R_{1}}{c}\right) \\
& \times \operatorname{rect}\left(\frac{f_{a} R_{B 1}}{\sqrt{f_{a m}^{2}-f_{a}^{2}}}\right) \exp \left(-j \frac{2 \pi f_{a} x_{l}}{v}\right) \\
& \times \exp \left(-j \frac{2 \pi R_{B q} \sqrt{f_{a m}^{2}-f_{a}^{2}}}{v}\right) \mathbf{W}_{n}^{H} z_{a} \\
& =\operatorname{sinc}\left(t_{k}-\frac{2 R_{1}}{c}\right) \operatorname{rect}\left(\frac{f_{a} R_{B 1}}{\sqrt{f_{a m}^{2}-f_{a}^{2}}}\right) \\
& \times \exp \left(-j \frac{2 \pi f_{a} x_{l}}{v}\right) \\
& \times \exp \left(-j \frac{2 \pi R_{B q} \sqrt{f_{a m}^{2}-f_{a}^{2}}}{v}\right) \mathbf{H}_{n} .
\end{aligned}
$$

After this, the resolving azimuth ambiguity operation is finished.

According to array signal theory, the Doppler ambiguity time $K$ must satisfy the following formula: $K \leq P$. To the member of azimuth subaperture $P$, it can make one restriction direction at most and the other $P-1$ is zero, so only $P$ time's ambiguity can be resolved on azimuth Doppler dimension. With the same principle, only $Q$ time ambiguity can be resolved on azimuth Doppler dimension.

After resolving the azimuth ambiguity, the signal is combined according to the Doppler spectrum. The range resolving ambiguity is followed. 
3.2. Resolving Range Ambiguity. According to (8), the range steering vector and the azimuth matching function are concerned. We expressed matrix vector $\mathbf{z}_{b}\left(R_{l}\right)$ as follows:

$$
\begin{aligned}
\mathbf{z}_{a}\left(R_{l}\right)= & {\left[\exp \left(-j \frac{2 \pi R_{B 1} \sqrt{f_{a m}^{2}-f_{a}^{2}}}{v}\right), \ldots,\right.} \\
& \left.\exp \left(-j \frac{2 \pi R_{B Q} \sqrt{f_{a m}^{2}-f_{a}^{2}}}{v}\right)\right]^{T} .
\end{aligned}
$$

It is assumed that the vector matrix is $\mathbf{W}_{\mathrm{Q} \times L}$ and the $m$ th column weighting vector is shown as:

$$
\mathbf{W}_{m}=\left[w_{1, m}, \ldots, w_{q, m}, \ldots, w_{\mathrm{Q}, m}\right]^{T}
$$

It means that the corresponding downlooking angle and azimuth angle position is 1 and other ambiguities are zero. Consider

$$
\mathbf{W}_{m}^{H} z_{b}=\mathbf{H}_{m}
$$

where ()$^{H}$ expresses matrix conjugate transpose operator and $\mathbf{H}_{m}=\left[h_{1 m}, \ldots, h_{L m}\right]^{T}, m=1, \ldots, L$, when $l=m, h_{l m}=1$. Consider

$$
\mathbf{W}_{m}=\left(\mathbf{H}_{m} z_{b}^{+}\right)^{H}
$$

where ()$^{+}$denotes the pseudoinverse operation, $z_{b}^{+}=$ $\operatorname{pinv}\left(z_{b}\right)$. Consider

$$
\begin{aligned}
\mathbf{S}^{\prime}= & {\left[s_{1}, \ldots, s_{\mathrm{Q}}\right]^{T} } \\
= & \operatorname{sinc}\left(t_{k}-\frac{2 R_{1}}{c}\right) \operatorname{rect}\left(\frac{f_{a} R_{B 1}}{\sqrt{f_{a m}^{2}-f_{a}^{2}}}\right) \exp \left(-j \frac{2 \pi f_{a} x_{l}}{v}\right) \\
& \times \exp \left(-j \frac{2 \pi R_{B q} \sqrt{f_{a m}^{2}-f_{a}^{2}}}{v}\right) z_{b} .
\end{aligned}
$$

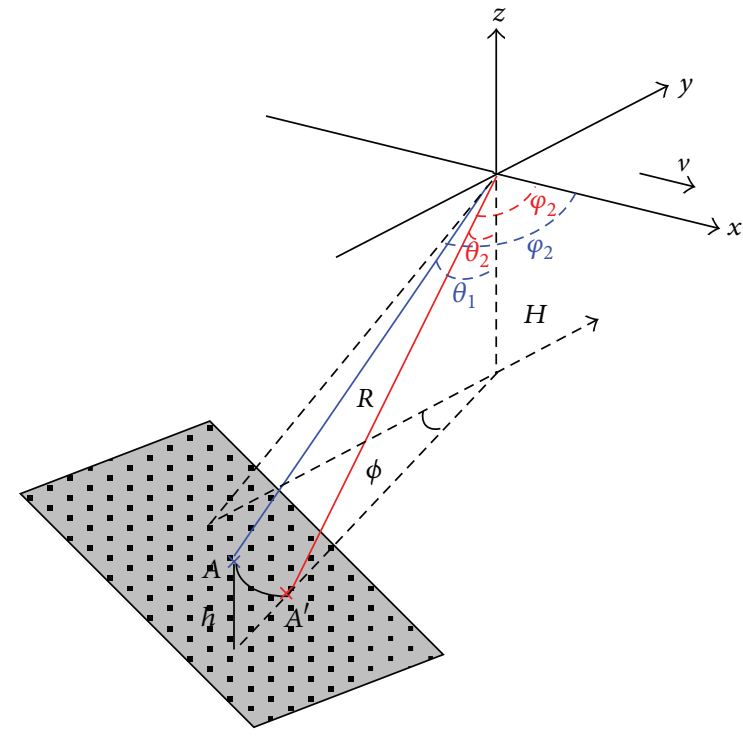

FIGURE 4: The geometry of terrain height variance.

Utilizing the weighting vector to resolve the ambiguity, we can gain

$$
\begin{aligned}
\mathbf{S}^{\prime \prime}=\mathbf{W}_{m}^{H} \mathbf{S}^{\prime}= & \operatorname{sinc}\left(t_{k}-\frac{2 R_{1}}{c}\right) \\
& \times \operatorname{rect}\left(\frac{f_{a} R_{B q}}{\sqrt{f_{a m}^{2}-f_{a}^{2}}}\right) \exp \left(-j \frac{2 \pi f_{a} x_{l}}{v}\right) \\
& \times \exp \left(-j \frac{2 \pi R_{B q} \sqrt{f_{a m}^{2}-f_{a}^{2}}}{v}\right) \mathbf{W}_{m}^{H} z_{b} \\
= & \operatorname{sinc}\left(t_{k}-\frac{2 R_{1}}{c}\right) \operatorname{rect}\left(\frac{f_{a} R_{B q}}{\sqrt{f_{a m}^{2}-f_{a}^{2}}}\right) \\
& \times \exp \left(-j \frac{2 \pi f_{a} x_{l}}{v}\right) .
\end{aligned}
$$

At this time, the range ambiguity was resolved completely. The azimuth was finished when range was resolved. Then after the azimuth inverse FFT (IFFT), we can finish the azimuth pulse compression.

For different point target slant distance $R_{l}$, the subpulse time delay should be added. For the $l$ th subswath, $s^{\prime \prime}\left(R_{l}\right)$ added the time delay $\Delta T_{l}=T_{p_{1}}+\cdots+T_{p_{l-1}}$ to the range envelope, which make the real position of scatter point $\left(x_{l}, y_{l}, z_{l}\right)$ was get. Then the whole wide swath signal was obtained.

3.3. Impact of Terrain Height Variance. Forenamed static weighting vector is based on the assumption that the terrain is flat. Here we can analyze the impact of terrain height variance on the weighting vector. 


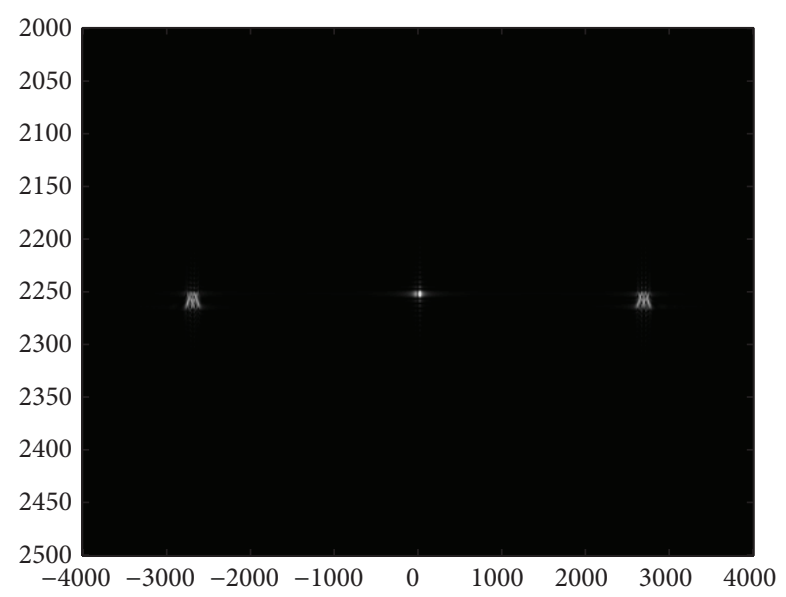

(a) The image with $2 \mathrm{D}$ ambiguity

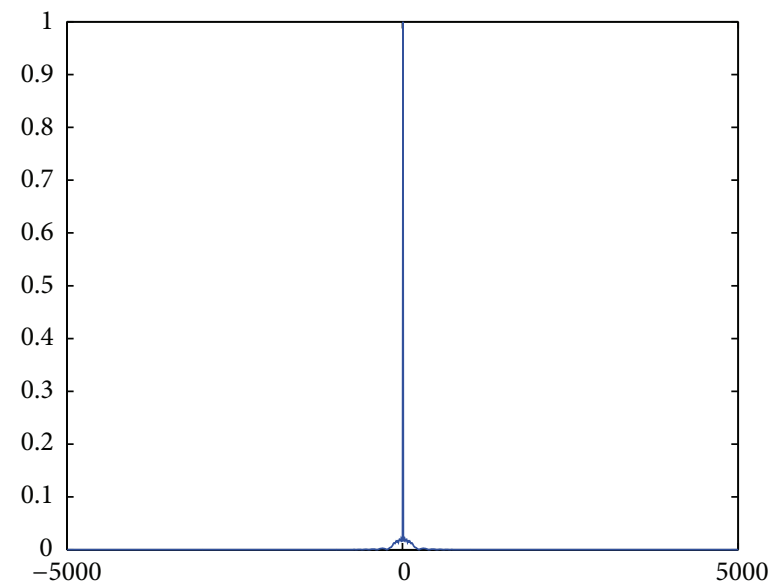

(c) Azimuth profile of far scatter point

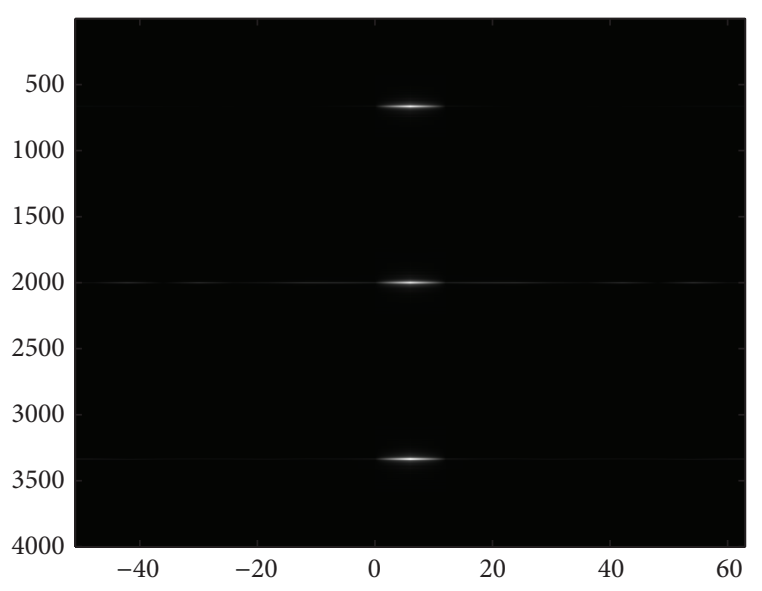

(b) The image after two-dimensional ambiguity resolving

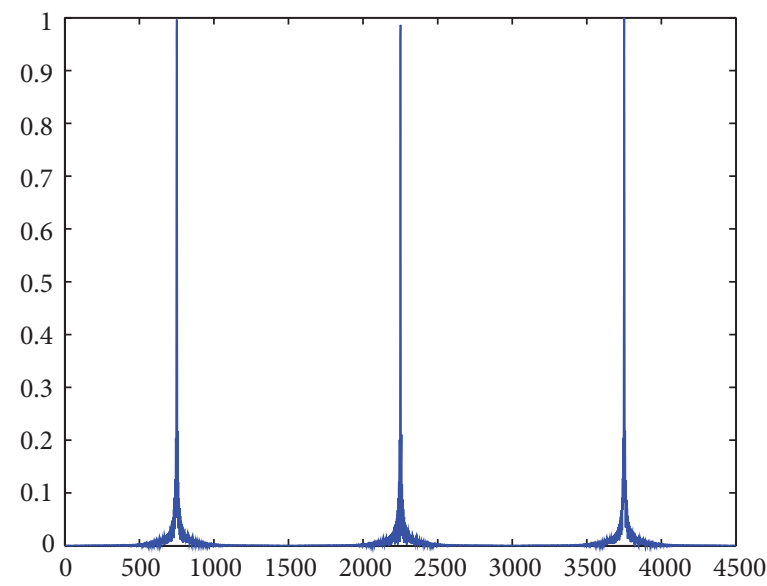

(d) Range profile of middle azimuth cell

FIGURE 5: The image of joint two-dimensional processing.

As shown in Figure 4, there is a point $A$ with the downlooking angle $\theta_{1}$ and azimuth angle $\varphi_{1}$; we assume there is another point $A^{\prime}$ with a height difference $h$ between $A$, so the phase difference of the weighting vector pointing to $A$ and $A^{\prime}$ is

$$
\Delta \Phi=\frac{2 \pi d_{1}\left(\cos \varphi_{1}-\cos \varphi_{2}\right)}{\lambda}-\frac{2 \pi d_{2}\left(\sin \theta_{1}-\sin \theta_{2}\right)}{\lambda},
$$

where $\theta_{2}, \varphi_{2}$ are the downlooking angle and azimuth angle in the position of $A^{\prime}$. From the geometry configuration of Figure 4, we can get that $\cos \varphi_{1}=\sin \theta_{1} \sin \phi$ and $\cos \varphi_{2}=$ $\sin \theta_{2} \sin \phi$, so the phase difference can be written as

$$
\begin{aligned}
\Delta \Phi & =\frac{2 \pi\left(\sin \theta_{1}-\sin \theta_{2}\right)\left(d_{1} \sin \phi-d_{2}\right)}{\lambda} \\
& =\frac{2 \pi\left(d_{1} \sin \phi-d_{2}\right)\left(\sqrt{R^{2}-(H-h)^{2}}-\sqrt{R^{2}-H^{2}}\right)}{\lambda R} .
\end{aligned}
$$

TABLE 1: Phase difference in the terrain with different height.

\begin{tabular}{lccc}
\hline Height $h(\mathrm{~m})$ & $\begin{array}{c}\text { Phase difference } \\
\Delta \Phi(\mathrm{rad})\end{array}$ & $\begin{array}{c}\text { Remained } \\
\text { clutter }(\mathrm{dB})\end{array}$ & Performance \\
\hline 352 & $-\pi / 32$ & -20.2 & Effective \\
940 & $-\pi / 12$ & -11.7 & Worse \\
1408 & $-\pi / 8$ & -8.2 & Much worse \\
3735 & $-\pi / 3$ & 0 & Ineffective \\
\hline
\end{tabular}

The clutter which remained caused by the phase difference is

$$
20 \log 10[1-\exp (j \Delta \Phi)]
$$

With the simulating parameter of $H=700 \mathrm{~km}, R=$ $880 \mathrm{~km}, \lambda=0.03 \mathrm{~m}, d_{1}=1 \mathrm{~m}$, and $d_{2}=0.8 \mathrm{~m}$, we get that the simulation result of the clutter which remained varied with the height difference shown in Table 1.

From Table 1 we can see that if the change of terrain height is below $1 \mathrm{~km}$, our proposed method can still be effective. 
TABLE 2: Simulation parameters.

\begin{tabular}{lc}
\hline Parameters & Value \\
\hline Height of satellite $H(\mathrm{~km})$ & 700 \\
Satellite velocity $v(\mathrm{~m} / \mathrm{s})$ & 7200 \\
Carrier frequency $f_{c}(\mathrm{GHz})$ & 2 \\
System PRF $(\mathrm{Hz})$ & 1200 \\
Stepped-frequency signal band width $B(\mathrm{MHz})$ & 100 \\
Transmitted pulse width $T_{\Delta}(\mu \mathrm{s})$ & 30 \\
Azimuth subaperture length $d_{1}(\mathrm{~m})$ & 4 \\
Elevation subaperture height $d_{2}(\mathrm{~m})$ & 0.8 \\
& $(0,42783.1,0)$ \\
Coordinates of the three scatter points $(\mathrm{m})$ & $(0,36703.5,0)$ \\
& $(0,39831.0,0)$ \\
\hline
\end{tabular}

\section{Simulation Experiment}

To validate the proposed approach, a simulation experiment on point targets is carried out. The main system parameters are listed in Table 2. In this simulation, the whole antenna aperture was divided into $3 \times 3$ subapertures.

It is supposed that these scatter points have the same backscattering coefficient. Through calculation one can get $R_{1}=c T_{\Delta} / 2+R_{2}=c T_{\Delta}+R_{3}$, so the three scatter points are range-ambiguous with each other. The Doppler band width is $B_{a}=2 v / D=3600 \mathrm{~Hz}$ and the azimuth sample frequency is $1200 \mathrm{~Hz}$, which makes the azimuth Doppler spectrum ambiguous with three times. Figure 5(a) shows the image of an original single channel. The azimuth and the range both have ambiguity. Range ambiguity makes three scatter points with different range positions mixed together, so only one point can be seen in Figure 5(a); because the system Doppler ambiguity three times, three focused points can be seen along azimuth. Both sides were ambiguity points and their energy was smaller than the center point obviously. Through the joint two-dimensional ambiguity resolving method proposed in this paper, the unambiguity image can be obtained as shown in Figure 5(b). After twodimensional ambiguity resolving, the scatter points were located in their real positions. Figure 5(c) is azimuth profile of the far scatter point. In the figure, the standard sinc function was obtained after the azimuth pulse compressed, which shows the unambiguous Doppler spectrum was recovered by the joint two-dimensional processing. Figure 5(d) is range profile of the three range points. From the figure, one can note that the range ambiguity is also resolved.

\section{Conclusion}

In this paper, a novel joint two-dimensional ambiguity resolving method based on space-time filtering was proposed, which can resolve the range and azimuth ambiguity of MIMO-SAR for high-resolution and wide swath imaging. This method does not use any approximate operation, which can finish resolving ambiguity and two dimensions focused instantaneously. Imaging results of the simulated point target validate the proposed approach. Because this method does not depend on SAR system work mode, it has certain general use.

\section{Conflict of Interests}

The authors declare that there is no conflict of interests regarding the publication of this paper.

\section{Acknowledgments}

This work was supported by the National Natural Science Foundation of China under Grant 61201433 and supported by Program for Young Talents of Science and Technology in Universities of Inner Mongolia Autonomous Region (NJYT14-B09).

\section{References}

[1] G. Krieger, N. Gebert, M. Younis, and A. Moreira, "Advanced synthetic aperture radar based on digital beamforming and waveform diversity," in Proceedings of the IEEE Radar Conference (RADAR '08), pp. 767-772, Rome, Italy, May 2008.

[2] A. Ossowska, J. H. Kim, and W. Wiesbeck, "Modeling of nonidealities in receiver front-end for a simulation of multistatic SAR system," in Proceedings of the 4th European Radar Conference (EURAD '07), pp. 13-16, Munich, Germany, October 2007.

[3] G. Krieger, N. Gebert, and A. Moreira, "Multidimensional waveform encoding: a new digital beamforming technique for synthetic aperture radar remote sensing," IEEE Transactions on Geoscience and Remote Sensing, vol. 46, no. 1, pp. 31-46, 2008.

[4] W. Q. Wang, "Space-time coding MIMO-OFDM SAR for high-resolution imaging," IEEE Transactions on Geoscience and Remote Sensing, vol. 49, no. 8, pp. 3094-3104, 2011.

[5] W. Wang, "Mitigating range ambiguities in high PRF SAR with OFDM waveform diversity," IEEE Geoscience and Remote Sensing Letters, vol. 10, no. 1, pp. 101-105, 2013.

[6] P. Huang and W. Xu, "A new spaceborne burst synthetic aperture radar imaging mode for wide swath coverage," Remote Sensing, vol. 6, no. 1, pp. 801-814, 2014.

[7] P. Huang, S. Li, and W. Xu, "Investigation on full-aperture on multichannel azimuth data processing in TOPS," IEEE Geoscience and Remote Sensing Letters, vol. 4, no. 11, pp. 728732, 2014.

[8] G. D. Callaghan and I. D. Longstaff, "Wide-swath space-borne SAR and range ambiguity," in Proceedings of the IEEE Radar Conference, pp. 248-252, Edinburgh, Scotland, October 1997.

[9] G. D. Callaghan and I. D. Longstaff, "Wide-swath space-borne SAR using a quad-element array," IEE Proceedings: Radar, Sonar and Navigation, vol. 146, no. 3, pp. 159-165, 1999.

[10] G. Krieger, N. Gebert, and A. Moreira, "Unambiguous SAR signal reconstruction from nonuniform displaced phase center sampling," IEEE Geoscience and Remote Sensing Letters, vol. 1, no. 4, pp. 260-264, 2004.

[11] G. Krieger, N. Gebert, and A. Moreira, "SAR signal reconstruction from non-uniform displaced phase centre sampling," in Proceedings of the IEEE International Geoscience and Remote Sensing Symposium Proceedings: Science for Society: Exploring and Managing a Changing Planet (IGARSS '04), vol. 3, pp. 17631766, Anchorage, AK, USA, September 2004. 
[12] N. Gebert, G. Krieger, and A. Moreira, "SAR signal reconstruction from non-uniform displaced phase centre sampling in the presence of perturbations," in Proceedings of the IEEE International Geoscience and Remote Sensing Symposium (IGARSS '05), pp. 1034-1037, Seoul, Korea, July 2005.

[13] P. Huang, W. Xu, and W. Qi, "Two dimension digital beamforming pre processing in multibeam Scansar," Progress in Electromagnetics Research, vol. 136, pp. 495-508, 2013. 

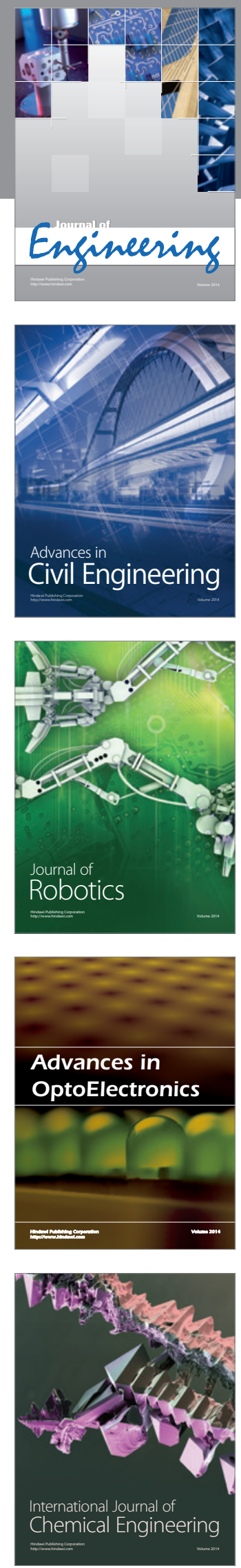

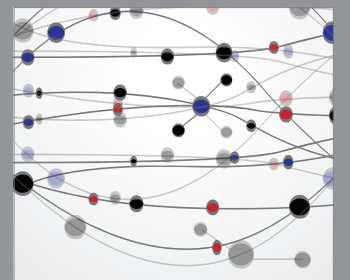

The Scientific World Journal
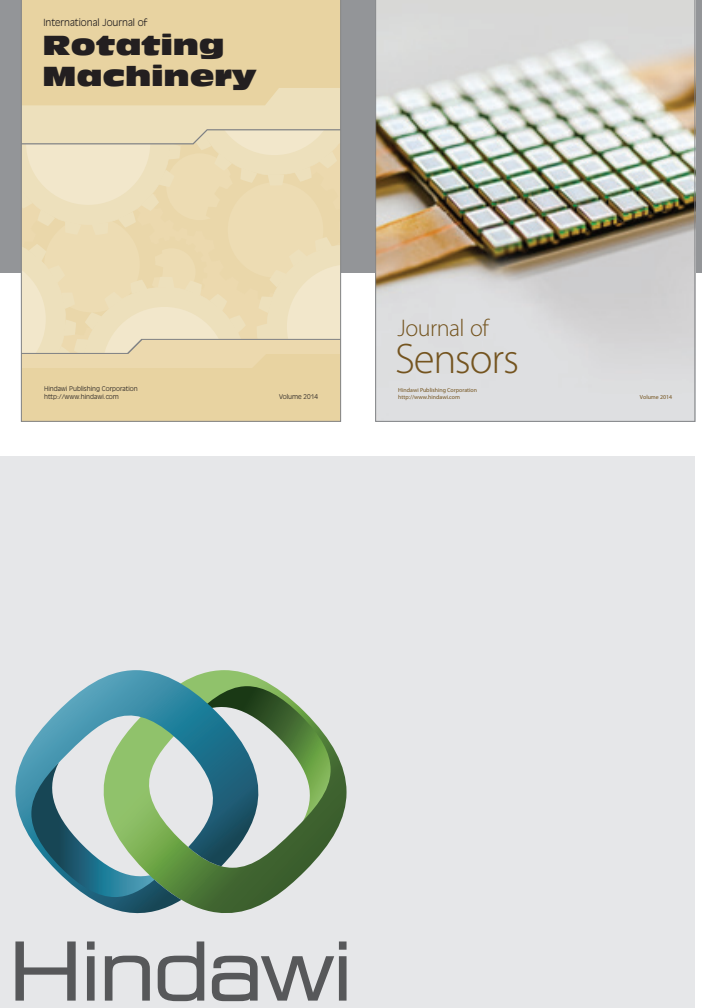

Submit your manuscripts at http://www.hindawi.com
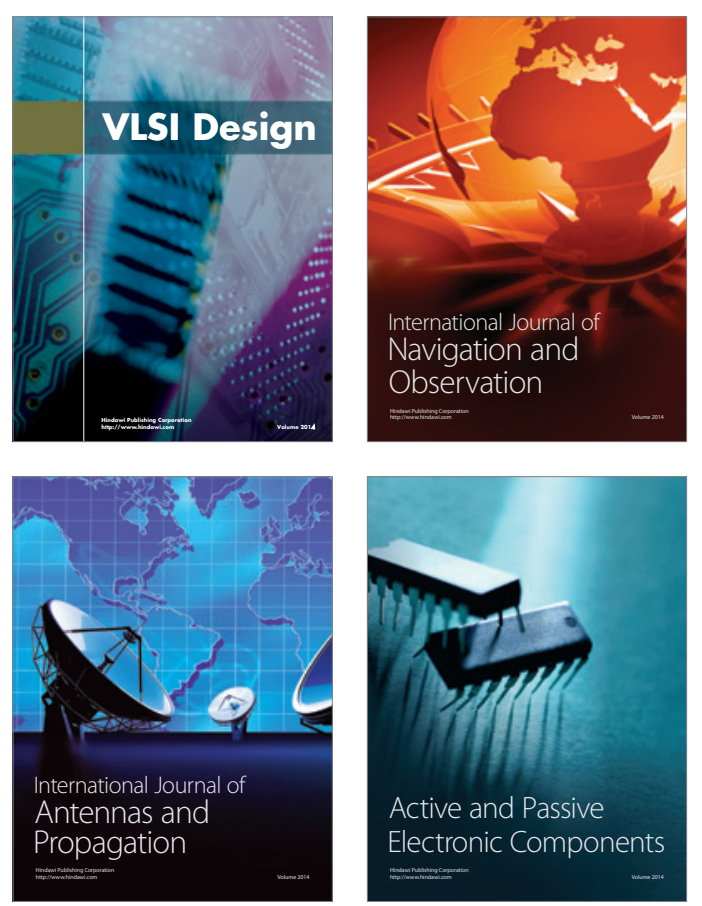
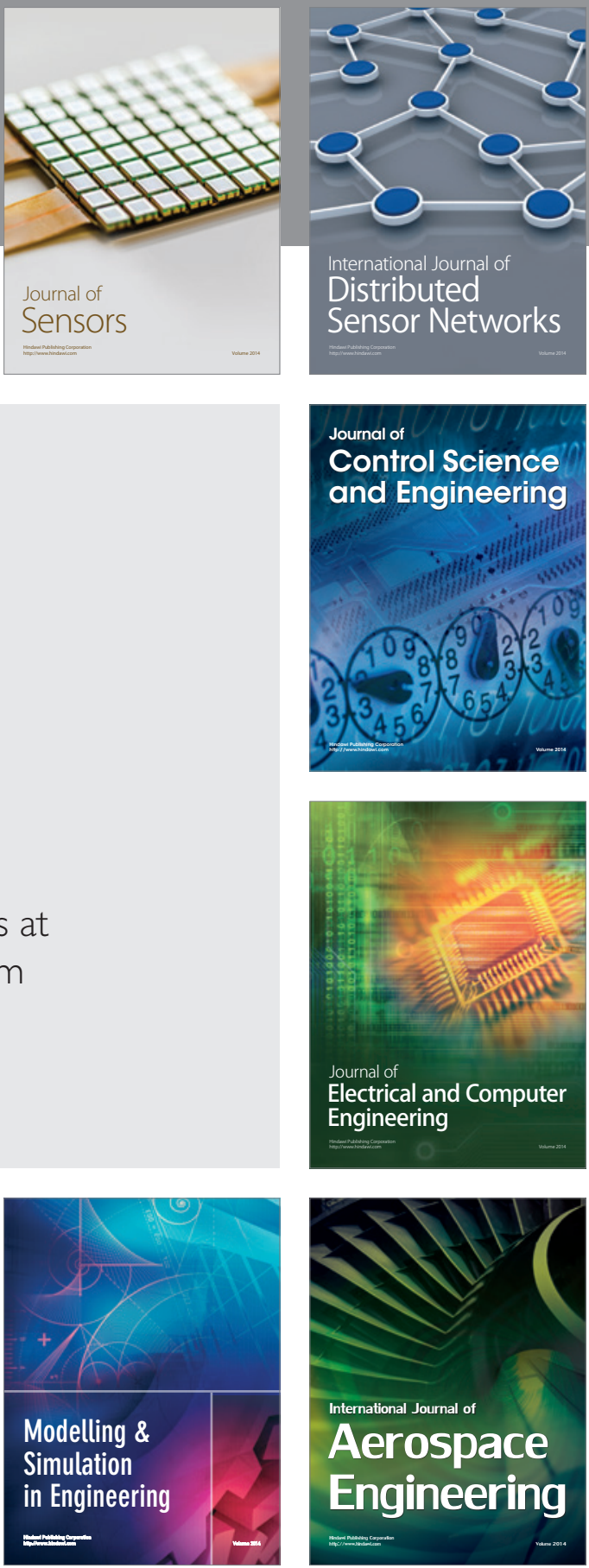

Journal of

Control Science

and Engineering
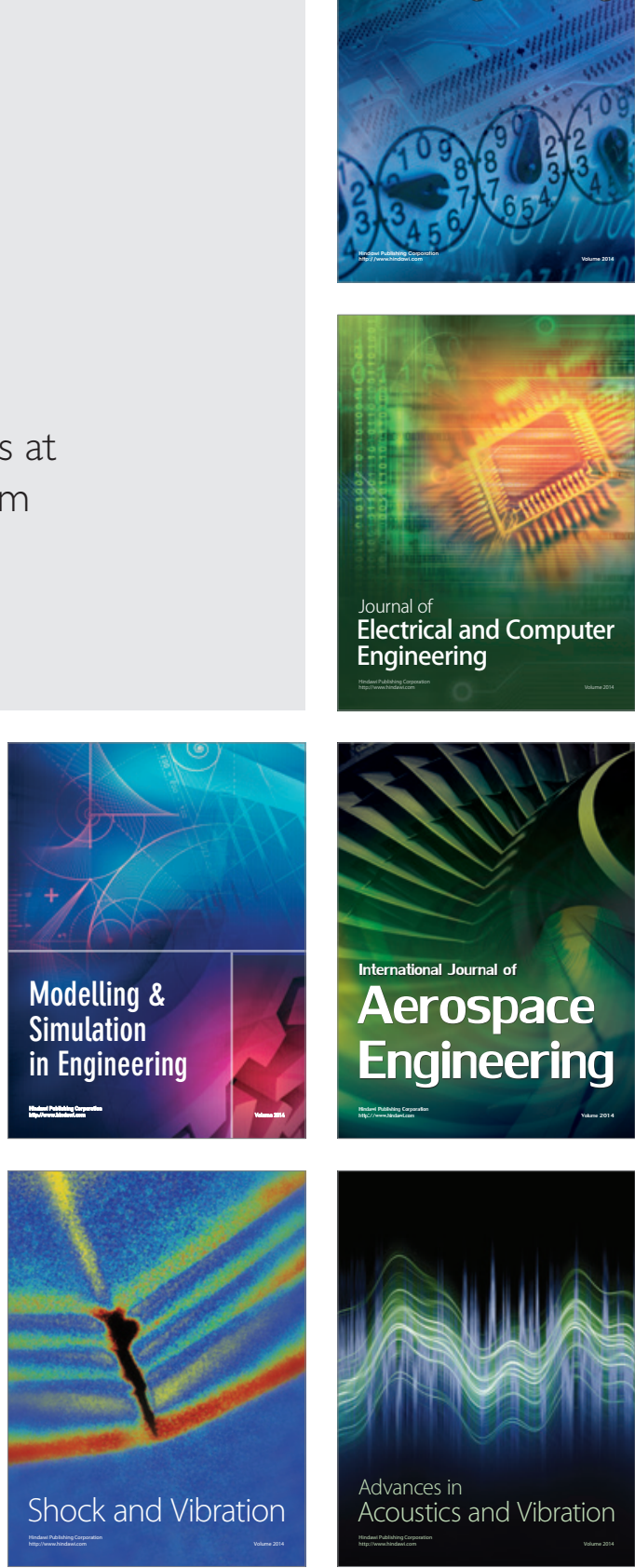